\title{
Factors influencing food waste - preliminary results from the national children's \& teen's food surveys
}

There is a growing body of research into "sustainable diets" which is a diet with low environmental impact, which contributes to food and nutrition security and a healthy life for present and future generations ${ }^{(1)}$. An important aspect of achieving a sustainable diet is a reduction in food waste ${ }^{(2)}$. The current study aimed to determine the factors influencing food waste using data collected during the National Children's and Teens Food Surveys (NCFSII and NTFSII) ${ }^{(3)}$.

The NCFSII and NTFSII were two cross-sectional food consumption surveys carried out between 2017 and 2020 in the Republic of Ireland. The sample included 600 children aged 5-12 years and 428 teenagers aged 13-18 years. The sample was representative of the Irish population with respect to age, sex, and urban/rural location according to the 2016 Irish census (CSO, 2016). Parents/guardians of participants were asked to complete a health and lifestyle questionnaire which included questions on food waste. Data was sought pertaining to the amount of food wasted by the household each week i.e. 'hardly any', 'less than 10\%', 'between 10-25\%', or 'greater than 25\%' for six different food categories; 1) All Foods, 2) Meat \& Fish, 3) Dairy Products, 4) Fruit \& Veg, 5) Bakery Products. Statistical analysis was carried out using the IBM statistical software package SPSS version 26. Chi-squared tests explored the relationship between the quantity of food wasted for each food category and demographic characteristics of participants including the location of their household, social class and frequency of grocery shopping.

Significant differences were observed between the amount of 'all food' wasted and the location of the participants household ( $p=0.001$ ); with only $13 \%$ of those living rurally in comparison to $52 \%$ of those in urban locations reporting that they waste between $10-25 \%$ of 'all food'. Preliminary results also suggest that frequency of grocery shop was a significant predictor of the amount of food wasted by a household with less food waste associated with less frequent or monthly grocery shopping $(p=0.01)$. Social class did not prove to be a determinant of food waste in this cohort when considering 'all food', however for 'fruit \& veg' only, $2 \%$ of professionals versus $10 \%$ of unskilled participants were found to waste more than $25 \%(\mathrm{p}=0.001)$.

In conclusion, this preliminary analysis suggests that educating certain demographic groups e.g. lower social classes, particularly in urban locations on the impact of food waste on the environment may help the population to achieve a more sustainable diet for future generations.

\section{References}

1. FAO (2012) [Available at: http://www.fao.org/3/i3004e/i3004e.pdf]

2. Conrad et al. (2021) [Available at: https://www.ncbi.nlm.nih.gov/pmc/articles/PMC5905889/]

3. www.iuna.net 